\title{
Die Kompatibilităt curricularer und methodischer Modellierungen als notwendige Bedingung für Unterrichtsqualität
}

Jürgen Seifried, Karsten D. Wolf, Christina Kuber und Detlef Sembill

\section{Ausgangslage}

Die Forderung nach "handlungsorientiertem" Unterricht (nicht selten ist damit die Umsetzung von i.d.R. gemäßigten konstruktivistischen Konzepten gemeint) ist - zumindest in Präambeln von Rahmenlehrplänen und anlässlich von "Lehrproben“ - allgegenwärtig (siehe z.B. Beyen 2003). Stichprobenartige Einblicke in die Unterrichtspraxis verdeutlichen jedoch in erschreckender Regelmäßigkeit, dass sich Wunsch und Wirklichkeit - wie nicht selten im Bildungswesen - nahezu diametral gegenüberstehen. Dies gilt sowohl für den allgemeinbildenden Bereich - mal wird hier der Physikunterricht als kreidelastiger "Demonstrationsunterricht" gekennzeichnet (Baumert \& Köller 2000), mal wird aus Österreich berichtet, dass der durchschnittliche zeitliche Anteil des Projektlernens etwa $0,5 \%$ der gesamten Unterrichtszeit beträgt (Petri 1991, S. 78) - als auch für den beruflichen Sektor. Wie beispielsweise eine jüngst abgeschlossene Befragung von 177 Lehrkräften und uber 1.400 Schülern aus dem kaufmännisch-verwaltenden Bereich zeigt, dominiert in der schulischen Praxis nach wie vor der fragend-entwickelnde Frontalunterricht; handlungsorientierte Lehr-LernArrangements nehmen eine eher ergänzende Funktion ein (Pătzold, Klusmeyer, Wingels \& Lang 2003, S. $141 \mathrm{ff}$.). ${ }^{1}$ So gaben in der genannten Studie knapp $47 \%$ der befragten Schüler an, noch nie mit Projektunterricht in Kontakt gekommen zu sein, die Hälfte der Probanden hatte keine Erfahrung mit Erkundungen und über drei Viertel der Lernenden warteten im kaufmännischen. Unterricht vergebens auf den Einsatz von Planspielen.

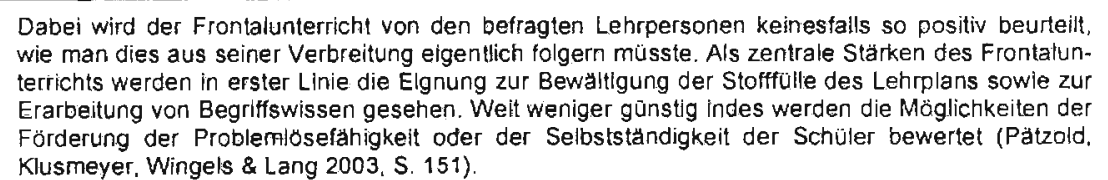


Nun könnte man die Kritik an der Dominanz der fragend-entwickelnden Vorgehensweise als unbedeutend abtun und aktuelle Erkenntnisse der Hirnforschung (zur "Brackenbildung zwischen Hirnforschung und Didaktik" siehe Friedrich \& Preiß 2003) weiterhin ignorieren, wenn nicht internationale Schulleistungsvergleiche zeigen würden, dass die hierzulande verfolgte Art und Weise des Unterrichtens lediglich suboptimale Lernergebnisse zu Tage fordert (Baumert, Bos \& Lehmann 2000; Baumert, Klieme, Neubrand, Prenzel, Schiefele, Schneider, Stanat, Tillmann \& Weiss 2001). Nicht nur aus der Sicht des Qualifikations- oder Bildungscontrolling (Seeber 2000) ist die skizzierte Entwicklung daher kritisch zu hinterfragen. Es muss endlich der Versuch unternommen werden, neue, effolgversprechende Wege der Qualifizierung nicht nur zu propagieren, sondern diese breitenwirksam in die schulische und betriebliche Ausund Weiterbildungspraxis zu tragen.

Für den kaufmännischen Sektor hat die Frage der Optimierung von Lehr-LernProzessen insbesondere für das Rechnungswesen Gewicht, sieht sich dieses Kernelement kaufmännischer Bildung doch seit Jahren in der fachdidaktischen Diskussion (siehe z.B. Achtenhagen 1990; Preiß \& Tramm 1996; Sloane 1996; Preiß 1999; Schneider 2000; Reinisch \& Struve 2002; Seifried 2002, 2004a). Die vielfältigen Monita an der traditionellen Vorgehensweise sind vornehmlich an zwei Punkten festzumachen: Zum einen ist die Ausrichtung an der Bilanzmethode - das bis heute in der Unterrichtspraxis vorherrschende didaktische Modell - als problematisch zu kennzeichnen. Zum anderen zielt die Kritik auf die lehrenentrierte Ausgestaltung der Lehr-Lem-Prozesse ab. Die Beanstandungen richten sich dabei in erster Linie gegen eine in kleine Lernschritte gegliederte, stark lehrergesteuerte Vorgehensweise. Erschwerend kommt hinzu, dass die einzelnen Lehreinheiten weitgehend identisch aufgebaut und Methoden nur selten variiert werden. Ein von uns im Rahmen eines Forschungsprojekts (siehe Abschnitt 2) befragter Seminarlehrer äußert sich diesbezüglich wie folgt:

"Ich habe schon erkannt, dass der Rechnungswesenunterricht sehr schematisch ist, gerade im Bereich Buchführung. Der Schüler weiß wie der Buchungssatz lautet, aber wenn man hinterfragt, dann ist es inm eigentlich gar nicht mehr so geläufig: Warum ist das 50 ? ... Wichtig ist die Abkehr vom schematischen Denken, das sich gerade imm Ber

Im vorliegenden Beitrag wird unter Rückbezug auf die genannten Schwächen der herkömmlichen Qualifizierung eine didaktische Alternative vorgestellt, mit der es gelingen kann, die skizzierten Monita zu überwinden. Aus unterrichtsmethodischer Sicht bieten Lehr-Lern-Arrangements mit hohen Anteilen selbstorganisierten Lernens
(SoLe) nach Sembill $(1992 a, 1999,2000)$ einen geeigneten Rahmen, um eine Ausweitung der Handlungsorientierung zu realisieren (siehe Abbildung 1, Entwicklungspfad 1).

Abb. 1: Entwicklungsmöglichkeiten des Rechnungswesenunterrichts

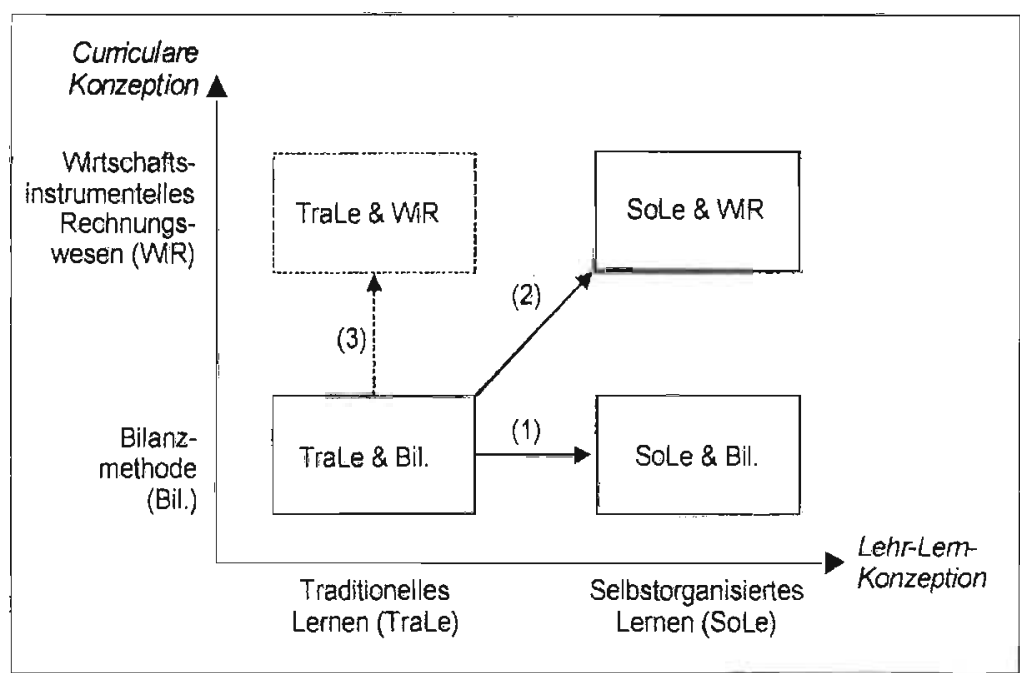

Dieses Ansinnen ist besonders dann erfolgreich, wenn aus curricularer Perspektive ein mit den Anforderungen des Selbstorganisierten Lernens kompatibler Ansatz herangezogen wird. Mit dem Göttinger Ansatz der Didaktik des wirtschaftsinstrumentellen Rechnungswesens (Preiß \& Tramm 1996, Preiß 1999, siehe auch die Beiträge von Preiß und Tramm in diesem Band) liegt mittlerweile eine fachdidaktische Alternative vor, in der die Bilanz als formales Referenzmodell durch ein inhaltliches Referenzmodell (das "Allgemeine Unternehmensmodeil") ersetzt wird und deren Grundprinzipien eine handlungsorientierte Unterrichtsgestaltung unterstützen. Insbesondere die Kombination des Selbstorganisierten Lernens mit der Didaktik des wirtschaftsinstrumentellen Rechnungswesens (siehe Abbildung 1, Entwicklungspfad 2) sollte daher eine vielversprechende Option darstellen, wenn man durch eine schüleraktive und selbstorganisationsoffene Gestaltung der Lehr-Lern-Prozesse eine Steigerung der Unterrichtsqualităt erreichen und gute Ergebnisse sowohl hinsichtlich kognitiver als auch motivational-emotionaler Erfolgskriterien erzielen möchte. Der ebenfalls denkbare Entwicklungspfad 3 (Kombination des fragend-entwickelnden Frontalunterrichts mit der Didaktik des wirtschaftsinstrumentellen Rechnungswesens) wurde an- 
gesichts der überzeugenden Befunde zum Selbstorganisierten Lernen ${ }^{1}$ sowie im Hinblick auf die prinzipiell als handlungsorientiert zu kennzeichnende Grundausrichtung des Göttinger Ansatzes im Rahmen der im Folgenden darzustellenden empirischen Untersuchung nicht weiter verfolgt.

\section{Konzeption der empirischen Untersuchung}

Ausgangspunkt der empirischen Untersuchung (siehe zusammenfassend Seifried 2004a) war die Frage, wie sich fachdidaktisch-curriculare Variationen (wirtschaftsinstrumentelles Rechnungswesen vs. Bilanzmethode) auf den Unterricht innerhalb einer selbstorganisationsoffenen Lernumgebung auswirken. Hierzu wurde in Zusammenarbeit mit Lehrkraften der Bamberger Berufsschule III eine entsprechende Lernumgebung gestaltet und evaluiert. An der auf ein Schuljahr (ca. 70 Unterrichtsstunden) ausgelegten quasi-experimentellen Feldstudie nahmen insgesamt drei Klassen der kaufmännischen Grundstufe (erstes Ausbildungsjahr) teil, davon dienten zwei Klassen als Experimental- und eine als Kontrollgruppe. Die beiden Experimentalgruppen wurden im SoLe-Arrangement unterrichtet, Unterschiede bestanden jedoch hinsichtlich der fachdidaktisch-curricularen Ausrichtung: Während eine Experimentalklasse herkömmlichen Rechnungswesenunterricht (Bilanzmethode, siehe Entwicklungspfad 1 in Abb. 1) erhielt, wurde die zweite nach den Prinzipien des wirtschaftsinstrumentellen Rechnungswesens unterrichtet (siehe Entwicklungspfad 2 in Abb. 1). Als Kontrollgruppe fungierte eine sowohl bezüglich der inhaltlichen Ausrichtung als auch der Unterrichtsmethode traditionell unterrichtete Klasse. Die drei Versuchsgruppen lassen sich somit wie folgt charakterisieren:

- Experimentalgruppe 1 ( $E G$ 1; $n=22$ ): Lehr-Lern-Methodik selbstorganisiert, Lerninhalte sowie Sequenzierung gemäß Bilanzmethode (SoLe + Bil.);

- Experimentalgruppe 2 ( $E G$ 2; $n=22$ ): Lehr-Lern-Methodik selbstorganisiert, Lerninhalte und ihre Sequenzierung nach den Grundsätzen des wirtschaftsinstrumentellen Rechnungswesens (SoLe + WiR)

- Kontrollgruppe ( $K G ; n=23$ ): Lehr-Lern-Methodik traditionell, d.h. überwiegend fragend-entwickelnder Frontalunterricht, Lerninhalte sowie Sequenzierung nach den Grundsătzen der Bilanzmethode (TraLe + Bil.).

In zwei von der Deutschen Forschungsgemeinschaft geförderten Untersuchungen zum Selbstorganisierten Lernen (Aktenzetchen Se 573/4-1, Se 573/4-2, Se 573/4-3) kennte nachgewiesen werden, dass Lerngrupper,
die in einem selbstorganisationsoffenen Lehr-Lern-Artangement lernten und arbeiteten, gegenüber Kontrollgruppen, die unter vergleichbaren bzw. teils deullich besseren Voraussetzungen in einer traditionellen Lernumgebung (TraLe) im Rahmen der "klassischen" Iehrerzentrierten Instruktion unterrichtet wurden, sowohl auf kogniliver als auch auf emotional-motivationaler Ebene überlegene Resultate erzieften (Sembill 1999. 2000. 2003a. 2004: Sembill, Wolf, Wutlke, Santjer \& Schumacher 1998; Wuttke 1999; Sembill, Schumacher, Wolf. Wutke \& San tjer-Schnabe; 2001).

\section{Zentrale empirische Befunde}

\subsection{Faktenwissen und Buchungskompetenz}

Zunächst gilt es zu prüfen, ob die Untersuchungsteilnehmer, die während eines Schuljahres in einer selbstorganisationsoffenen Lernumgebung lernten, ein zumindest vergleichbares Maß an Faktenwissen und Buchungskompetenz erwerben konnten wie ihre im Rahmen der herkömmlichen lehrerzentrierten Instruktion unterrichteten Mitschüler. Bei einer vergleichbaren methodischen Gestaltung des SoLeUnterrichts soltten, so die weiterführende Vermutung, Schüler, die nach den Grundsätzen des wirtschaftsinstrumentellen Rechnungswesens unterrichtet wurden, aufgrund konzeptionsimmanenter Vorzüge über bessere Fähigkeiten verfügen als Schüler, die einen an der Bilanzmethode orientierten Unterricht durchliefen.

Der Leistungsstand der beiden Experimentalgruppen wurde zu unterschiedlichen Zeitpunkten erhoben: (1) nach ca. einem Drittel, (2) kurz vor Ende des Untersuchungszeitraums sowie (3) ca. acht Wochen nach Beendigung des Rechnungswesenunterrichts. Gleiches gilt für die Kontrollklasse, allerdings mussten hier die abschließenden Tests aufgrund unterrichtsorganisatorischer Zwänge unmittelbar nach Ende des Rechnungswesenunterrichts durchgeführt werden, was zu einer Verfälschung - jeweils zugunsten der Kontrollklasse - beiträgt, weil im Unterschied zu den Experimentalklassen noch keine größeren Vergessenseffekte anzunehmen sind. Zur Messung der Performanz wurden lernzielorientierte Tests (LOT, Inhaltsgebiet Rechnungswesen) herangezogen, bei denen maximal 16 Punkte erreichbar waren. Abbildung 2 gibt einen ersten Überblick über Vorwissen und Leistungsentwicklung.

Zur statistischen Überprüfung möglicher Unterschiede zwischen den Vergleichsgruppen kam eine Kovarianzanalyse mit Messwiederholung zur Anwendung. ${ }^{1}$ Dabei dienen der lernzielorientierte Test als abhangige Variable, das Treatment und die Messwiederholung als Faktoren und das Vorwissen als Kovariate. Neben einem hoch signifikanten Einfluss des Vorwissens ergibt sich ein substanzieller Treatmenteffekt $\left(F=5.913 ; p=.004 ; \eta^{2}=162\right.$ ). Post-hoc-Tests zeigen, dass der Treatmenteffekt auf die Überlegenheit der Experimentalgruppe 2 sowohl gegenüber der Experimentalgruppe 1 als auch im Vergleich zur Kontrollgruppe zurückgeht. Die festgestell-

\footnotetext{
In der Expertiseforschiung wird insbesondere die Bedeutung des aufgaben- und bereichsspezifischen Vorwissens für die Leistungsentwicklung herausgestellt. Deshalb gilt es zu prüfen, ob ein Zusammen hang zwischen
diesem Faktor und der Zielgroßße vorliegt Dabei zeigt sich eine hoch signifikante Korrelation $\left(r=62^{* *}\right) z w i-$ schen dem Vorwissen im Bereich Rechnungswesen und dem gemithelten Resultat der lemzielorientierten Tests uber die drei Messzeitpunkte hinweg.
} 
ten Mittelwertunterschiede zwischen Experimentalgruppe 1 und Experimentalgruppe 2 sowie zwischen Experimentalgruppe 2 und Kontrollgruppe lassen sich jeweils zufallskritisch absichern $(p<.05) .{ }^{1}$ Darüber hinaus ist der Leistungsstand der Experimentaigruppe 1 und der Kontrollgruppe nahezu gleich (mittlere Differenz $=.05, p=$ 1.000). Unter Berücksichtigung des bereichsspezifischen Vorwissens besteht also kein Nachteil der Experimentalgruppe 1 gegenüber der Kontrollgruppe.

Abb. 2: Leistungsentwicklung der Vergleichsgruppen

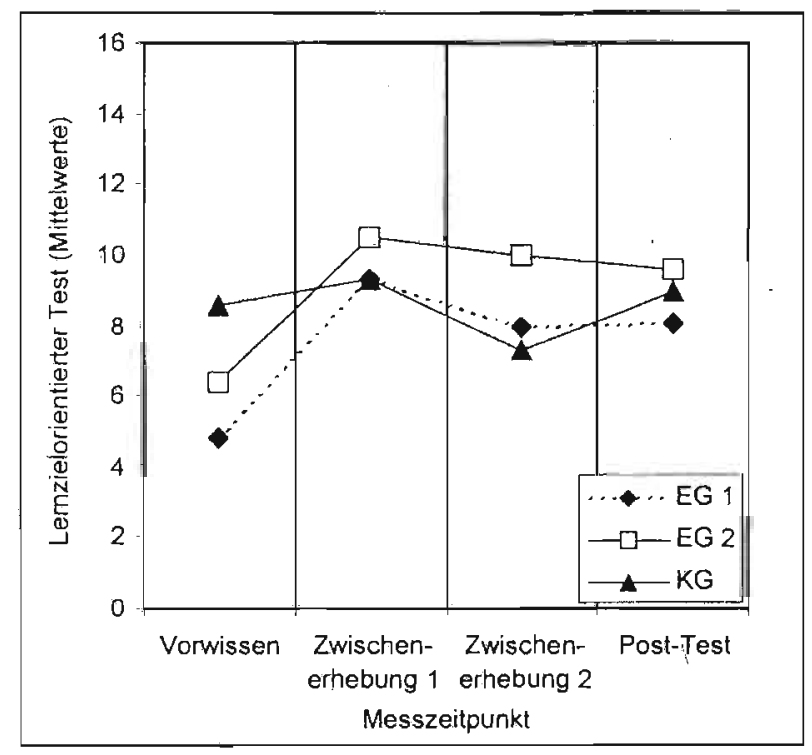

Das Ergebnis untermauert die Vermutung, dass SoLe-Schüler bezüglich der Fähigkeit, Faktenwissen zu reproduzieren und Buchungen vorzunehmen, einen (mindestens) vergleichbaren Leistungsstand erreichen wie Schüler, die in traditioneller Weise unterfichtet werden. Nach der Neutralisierung von Vorwissensunterschieden ergibt sich für Experimentalgruppe 2 ein signifikanter Vorteil gegenüber der Kontrollgruppe, während Experimentalgruppe 1 genauso gut abschneidet wie die TraLe-Klasse. Verstärkend kommen noch die nicht berücksichtigten, Jedoch wahrscheinlichen Effekte hinsichtlich fehlenden Vergessens der Kontrollgruppe hinzu (s.o.). Die zu Projektbeginn seitens der beteiligten Lehrkräfte geäußerte Befürchtung, dass das Lernen in Bei den durchgeführten Mehrfachvergleichen im Fall signifikanter Trealmenleffekte wird das beobachtele Sig-
nifikanzniveau jeweils mit Hilfe des BONFERRON-Verfahrens korrigiert, um eine Kumulierung des $a$-Fehlers zu nifikanzniveau jeweils mit Hilfe des BONFERRONL-Verahrens komigier, um eine Kumulierung des a-Fehlers
vermeiden. Diese Berichtigung führt zu eher konservativen Entscheidungen zugunsten der Nullhypothese. einer selbstorganisationsoffenen Lernumgebung für die Ausbildung von "weichen" Qualifikationen wie Teamfähigkeit und Kommunikationskompetenz einen geeigneten Rahmen biete, die Schüler mit der selbstständigen Aneignung von Faktenwissen/Buchungskompetenz jedoch überfordert seien, kann in Anbetracht der vorliegenden Resultate zurückgewiesen werden. Die Befunde unterstützen darüber hinaus auch die zweite Vermutung: Die SoLe-Klasse, die nach den Grundsätzen des wirtschaftsinstrumentellen Rechnungswesens unterrichtet wurde, erweist sich im Vergleich zu der Kombination des Selbstorganisierten Lernens mit der Bilanzmethode als überlegen (die Gegenüberstellung beider Experimentalgruppen ergibt einen signifikanten Unterschied zugunsten der Experimentalgruppe 2). Experimentalgruppe 2 verfügt gegenüber den beiden anderen Klassen somit über nennenswerte Vorteile, und zwar unabhängig davon, ob die Bilanzmethode mit Selbstorganisiertem Lernen oder mit lehrerzentrierter Instruktion kombiniert wurde.

\subsection{Problemlösekompetenz}

Der oben skiżzierte Vergleich der Leistungsentwicklung im Bereich Wissensreproduktion kann nicht ais das entscheidende Gütemaß für die Qualität von Lehr-LernProzessen gelten. Die Ergebnisse lernzielorientierter Tests, die Faktenwissen und Buchungskompetenz auf dem Niveau von Kammerabschlussprüfungen abfragen, liefern nur einen ersten Ansatzpunkt für die Beurteilung des Lernerfolgs. Als von zentraler Bedeutung erweist sich vielmehr die Entwicklung im Bereich der Problem/ösekompetenz. Dabei liegt der Fokus der Bemühungen auf der Förderung der Fähigkeit, in komplexen Situationen kompetent handeln zu können (Hacker 1992; Sembill 1992a; Mayer \& Wittrock 1996; Gruber, Mandl \& Renk| 2000).

Angesichts der bisherigen überaus positiven Erfahrungen mit der Lehr-LernKonzeption des Selbstorganisierten Lernens (s.o.) kann davon ausgegangen werden, dass die SoLe-Schüler im Vergleich zu den traditionell unterrichteten Schülern bessere Möglichkeiten vorfinden, ihre Problemlösekompetenz zu steigern. Darüber hinaus sollten insbesondere nach den Grundsätzen des wirtschaftsinstrumentellen Rechnungswesens unterrichtete Schüler hinsichtlich der bereichsspezifischen Problemlösekompetenz über einen Kompetenzvorsprung gegenüber jener Klasse verfügen, die in eíner Kombination der Bilanzmethode mit Selbstorganisiertem Lernen unterrichtet wurde. 
Die Erfassung der Problemlösekompetenz vollzieht sich in zwei Schritten (siehe ausführlich Sembill 1992b): Zum einen erfolgt die Auswertung der von den Schülern bearbeiteten Problemfälle hinsichtlich vier quantitativer Kategorien (Ist-Zustand, SollZustand, Maßnahmen, Handlungskontrolle), die anschließend zu einem Gesamtkriterium (Analytischer Idealtypus gewichtet, AITG) zusammengefasst werden. Zum anderen ist die fachinhaltliche Qualităt der Problemlösung zu bewerten. Dabei kommen die qualitativen Kriterien "deklaratives Wissen", "Logik", "Eirfolgsaussichten der Problemlösung" und "Wissensvernetzung" zur Anwendung

Die Analyse der Problemlösekompetenz weist für das Gesamtmaß „Analytischer Idealtypus gewichtet" auf eine Überlegenheit beider Experimentalklassen hin. Wie erinnerlich wurden die Kriterien zu drei Zeitpunkten (erste und zweite Zwischenerhebung sowie Post-Test) gemessen. Bei der ersten Zwischenerhebung verfügen Kontrollgruppe und Experimentalklasse 2 über vergleichbare Fähigkeiten, die jene der Schüler der Experimentalgruppe 1 übersteigen. Bel der zweiten Zwischenerhebung indes erweisen sich die Versuchspersonen der beiden Experimentalklassen als die überlegenen Problemlöser, während die Entwicklung der Kontroliklasse stagniert. Beim Post-Test ist für alle Vergleichsgruppen ein Anstieg zu verzeichnen, für die Kontrollklasse allerdings auf einem geringeren Niveau. Abbildung 3 zeigt die Entwicklung des Gesamtmaßes AITG

\section{Abb. 3: Entwicklung der lerninhaltsspezifischen Problemlösekompetenz}

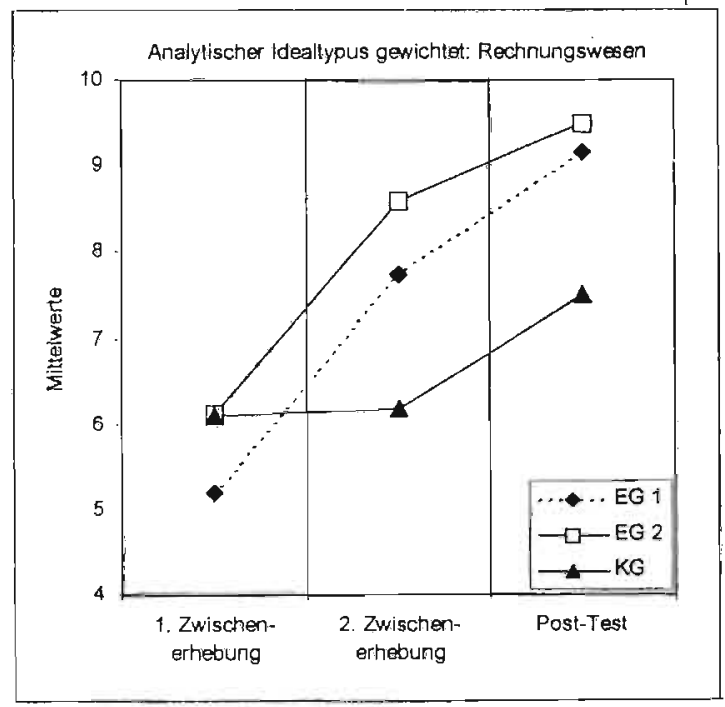

Um das Vorhandensein von Treatment-, Zeit- und Wechseiwirkungseffekten für den AITG und dessen quantitative Unterkategorien zu prüfen, kommt eine zweifaktorielle Kovarianzanalyse mit Messwiederholung zur Anwendung. ${ }^{1}$ Die Analyse ergibt zunăchst für die Variable "AITG" einen signifikanten Wechselwirkungseffekt gekoppelt mit einem substanziellen Treatment- und Zeiteffekt. Dieser Interaktionseffekt ist auf den im Vergleich zur Kontrollgruppe stärkeren Kompetenzzuwachs beider Experimentalgruppen zurückzuführen (siehe Abbildung 3). Bei dem durchgeführten Posthoc-Test stellen sich die beiden SoLe-Klassen im Vergleich zur Kontrollgruppe als bessere Problemlöser heraus, die Unterschiede sind jeweils (hoch) signifikant (Experimentalgruppe 1 vs. Kontrollgruppe: mittlere Differenz $=1.40, p=.029$; Experimentalgruppe 2 vs. Kontrollgruppe: mittlere Differenz $=1.57, p=.009$ ). Für die Kriterien "Logik" und "Wissensvernetzung" lassen sich erneut signifikante Interaktionseffekte feststellen. Bezüglich der genannten Kategorien weist insbesondere die Experimentalgruppe 2 einen Kompetenzzuwachs auf, wohingegen die Entwicklung in der Experimentalgruppe 1 sowie der Kontrollgruppe weniger günstig verläuft. Für die Kriterien "deklaratives Wissen" und "Erfolgsaussichten der Problemlösung" ergeben sich keine überzufälligen Effekte (siehe Abbildung 4).

Bei der Analyse der lerninhaltsspezifischen Problemlösekompetenz erweisen sich also die beiden Experimentalklassen hinsichtlich des quantitativen Gesamtmaßes AITG als (hoch) signifikant bessere Problemlöser. Bezüglich der qualitativen Kriterien indes ist eine Überlegenheit der Experimentalgruppe 2 erkennbar. Die berichteten Befunde der Kovarianzanalysen unterstreichen damit die Vermutung insbesondere der Uberlegenheit der SoLe-Klasse, die nach den Prinzipien des wirtschaftsinstrumentellen Rechnungswesens unterrichtet wurde. 
Abb 4 Entwicklung der lerninhaltsspezifischen Problemlösekompetenz (qualitative Kategorien)

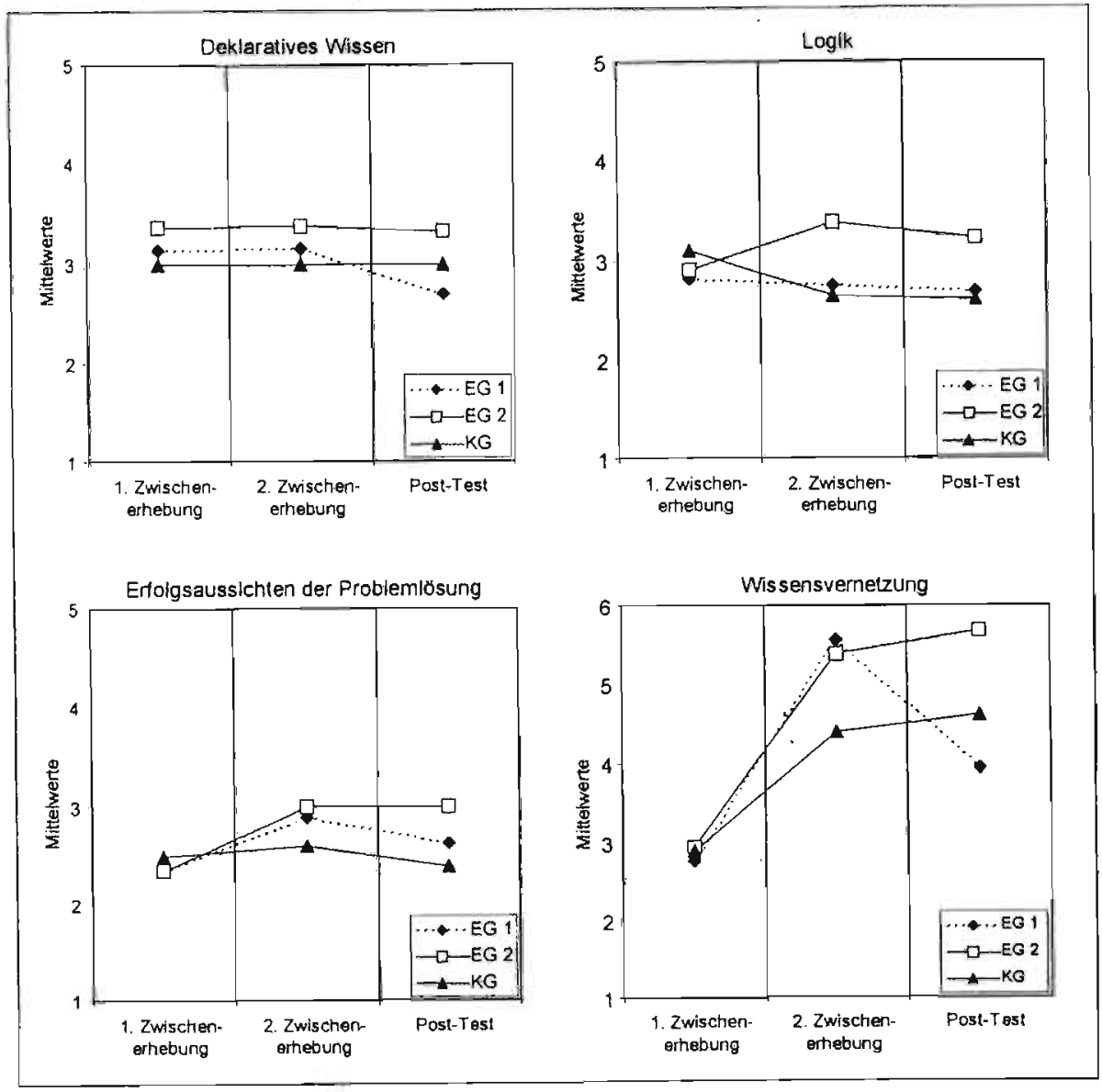

\subsection{Emotional-motivationale Erfolgskriterien}

Wie gezeigt, führt die Kombination des Selbstorganisierten Lernens mit der Didaktik des wirtschaftsinstrumentellen Rechnungswesens zu einer signifikanten Verbesserung der Lehr-Lern-Produkte. Ähnliches sollte auch für die Lehr-Lern-Prozesse gelten. Mit Hilfe des inhaltsspezifischen Motivationsfragebogens von Prenzel (1994) wurde überprüft, wie die Schüler den Rechnungswesenunterricht hinsichtlich emotio- nal-motivationaler Kriterien beurteilen. ${ }^{1}$ Diesbezüglich lässt sich vornehmlich festhalten, dass beide SoLe-Klassen im Vergleich zur TraLe-Klasse über Vorteile bezuglich der selbstbestimmten Motivationsarten (intrinsische Motivation und Interesse) verfügen (siehe Abbildung 5 sowie ausführlich Seifried 2004a, S. 167 ff.). Zu einem ähnlichen Ergebnis gelangen Sembill, Schumacher, Wolf, Wuttke \& Santjer-Schnabel (2001, S. 267 ff.) im Rahmen von Prozessanalysen Selbstorganisierten Lernens im Lerngebiet Personalwirtschaft. Damit bestätigt sich erneut, dass eine selbstorganisationsoffene Lernumgebung eine gute Möglichkeit darstellt, wenn Schüler sowohl hinsichtlich kognitiver als auch motivationaler Kriterien einen uberdurchschnittlichen Erfolg erzielen sollen. Die emotionalen Befunde korrespondieren den motivationalen Befunden in allen SoLe-Projekten. Die Emotionale Befindlichkeit bildet die Basis bedürfnisbefriedigender, motivierender Lern- und Arbeitsprozesse (Sembill 2003b, 2004).

Abb. 5: Entwicklung von intrinsischer Motivation und Interesse

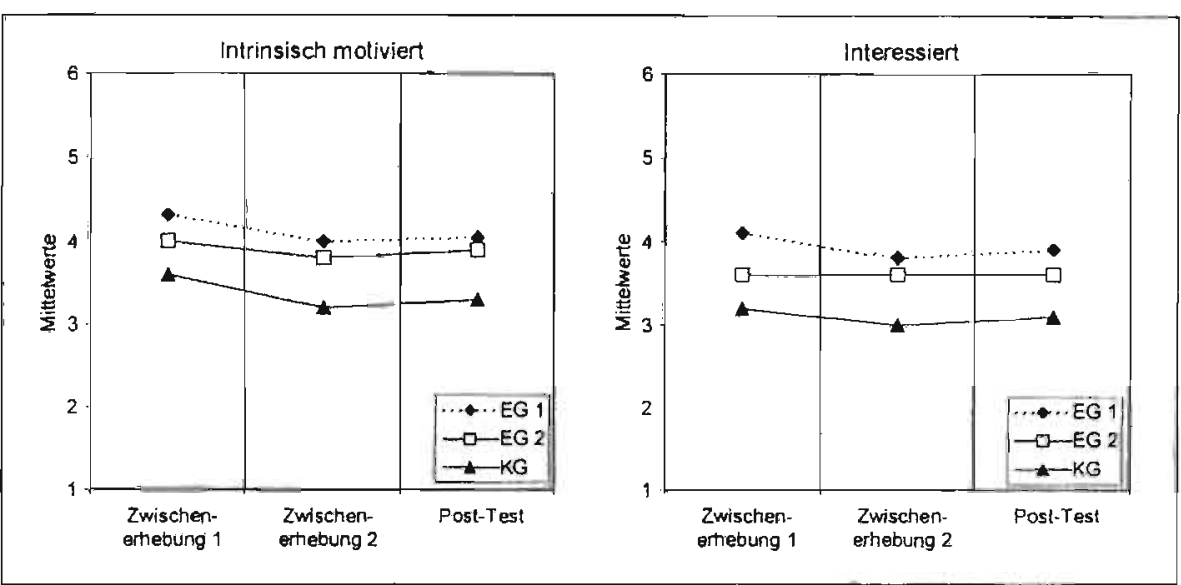

\subsection{Aktivitäten der am Unterricht Beteiligten}

Die vorstehenden Ausführungen haben gezeigt, dass die Orientierung an der LehrLern-Konzeption des Selbstorganisierten Lernens bei simultaner Ausrichtung an der Didaktik des wirtschaftsinstrumentellen Rechnungswesens zu einer signifikanten Verbesserung der Unterrichtsqualität führt. Im Folgenden sofl mit Blick auf die LehrNeben so genannten motivationsrelevanten Bedingungen werden positive und negative Emotionen
erfasst und sechs Motivationsausprägungen unterschieden (von amotiviert bis zu intrinsisch motiviert und interessiert) 
und Lernprozesse in den beiden SoLe-Klassen untersucht werden, welche Faktoren für die mehrfache Überlegenheit der Kombination von SoLe und wirtschaftsinstrumentellen Rechnungswesen verantwortlich zeichnen. Diesbezüglich ist zu vermuten, dass (a) beim Selbstorganisierten Lernen die eingeräumten Freirăume von den Lernenden insbesondere dann gewinnbringend genutzt werden können, wenn die fachdidaktisch-curriculare Modellierung die Schülerselbsttätigkeit in ausreichender Weise unterstützt und dass (b) die Ausrichtung der Lehr-Lern-Prozesse an der Didaktik des wirtschaftsinstrumentellen Rechnungswesens den Lehrpersonen die Erläuterung buchungsrelevanter Sachverhalte erleichtert.

\subsubsection{Analyse der Problemlöseprozesse in schülerzentrierten Arbeitsphasen}

Im Folgenden wird darüber berichtet, wie durch die Analyse von Schüler-SchülerInteraktionen während schülerzentrierter Arbeitsphasen Erkenntnisse über die Abläufe von Problemlöseprozesse beim Selbstorganisierten Lernen gewonnen werden können. ${ }^{1}$ In diesem Kontext ist von zentralem Interesse, ob zwischen den Problemlöseaktivitäten und dem Lernerfolg ein überzufälliger Zusammenhang besteht. Aufgrund des enormen Arbeitsaufwandes insbesondere der Verschriftlichung der Schüler-Schüler-Interaktionen war aus forschungspragmatischen Gründen eine Reduktion des umfangreichen Datenmaterials sowie eine Beschränkung auf ausgewählte Arbeitsgruppen unumgänglich. Die im Folgenden vorgestellten Befunde basieren auf der Analyse einer gegen Ende des Schuljahres angesiedelten Unterrichtseinheit, die vier Unterrichtsstunden umfasst. Gegenstand dieser Lernsequenz ist in beiden Vergleichsgruppen die Bearbeitung eines Beleggeschäftsgangs (vorbereitende Abschlussbuchungen) inklusive der grafischen Aufbereitung der Daten für einen Geschäftsbericht. Insbesondere die selbstständige Bearbeitung von Beleggeschäftsgängen sowie die Interpretation der Daten erscheinen geeignet, Informationen sowohl zur Buchungskompetenz als auch zum Verständnis ökonomischer Zusammenhänge zu gewinnen. Im Hinblick auf die Zielsetzung, die Auswirkungen fachdidak-

\footnotetext{
Bei schülerzentrierten Arbeitsphasen sehen sich Unterrichtspraktiker des Ofteren mit dem Problem konfrontiert, dass einzeine Schüler nur sporadisch (oder überhaupt nicht) bei der Bearbeitung der gestellten Aufträge mitmachen. Dieses als "Trittbrettfahren" bekannte Phänomen und die möglicherweise daraus resultierende Unzufriedenheit der Leistungsträger (siehe z.B. RENKL, GRUBER \& MANDL 1996; DANN, DIEGRITZ \& ROSENBUSCH 2002) wirken sich insbesondere dann negativ aus, wenn ein Großteil der Unterrichtszeit für schülerzentriertes Arbeiten aufgewendet wird (beim Selbstorganisierten Lernen immerhin ca. $65 \%$ der Unterrichtszeit). Im SoLe-Arrangement versucht man daher, durch obligatorische Präsentationen der Arbeitsergebnisse zu vermeiden, dass schülerzentrierte Arbeitsphasen in Nebenengagement (private Unterhaltung mit dem Nachbarn, Erledigen der Hausaufgaben für andere Fächer etc.) abgleiten. Zudem soll die wechselseitige Übernahme von Verantwortungsbereichen (z.B. Gruppenleitung, Gruppensprecher, Dokumentation der Arbeitsergebnisse) die einzelnen Schüter zur Mitarbeit motivieren.
}

tisch-curricularer Variationen im Rechnungswesenunterricht näher zu beleuchten, kommt dieser Einheit somit besonderes Interesse zu. Zudem wurde in einem zweiten Schritt die Auswertung auf jeweils zwei Lerngruppen pro Experimentalklasse beschränkt. In die Teilauswertung gehen somit insgesamt 17 Schülerinnen und ein Schüler ein.

Im Folgenden soll untersucht werden, ob für die ausgewählte Unterrichtseinheit zwischen den beobachtbaren Problemlöseaktivităten (Unterkategorien "Problemdefinition", "Problembearbeitung" und "Handlungskontrolle") und dem subjektiven Erleben des Unterrichts während der schülerzentrierten Arbeitsphase ein Zusammenhang besteht. Hierzu werden Rangkorrelationen (Kendalls tau) berechnet, da der Kolmogorov-Smirnov-Test auf Normalverteilung zeigt, dass sich die Werte der zu untersuchenden Variablen in der Stichprobe nicht normal verteilen. Tabelie 1 beinhaltet die für die Untersuchungsteilnehmer der Teilstichprobe über die Messzeitpunkte der ausgewählten schülerzentrierten Arbeitsphasen hinweg berechneten Korrelationen.

Tab. 1: Rangkorrelationen zwischen den Interaktionsdaten und der Lernleistung

\begin{tabular}{|c|c|c|c|c|c|c|c|c|c|c|c|c|}
\hline \multirow{3}{*}{$\begin{array}{l}\text { Häufigkeit } \\
\text { der }\end{array}$} & & & \multicolumn{10}{|c|}{ Lerninhaltsspezifische Problemłösekompetenz } \\
\hline & \multicolumn{2}{|c|}{ LOT } & \multicolumn{2}{|c|}{ AITG } & \multicolumn{2}{|c|}{$\begin{array}{c}\text { Deklaratives } \\
\text { Wissen }\end{array}$} & \multicolumn{2}{|c|}{$\begin{array}{l}\text { Logik der } \\
\text { Problemlösung }\end{array}$} & \multicolumn{2}{|c|}{$\begin{array}{l}\text { Efolgs- } \\
\text { aussichten }\end{array}$} & \multicolumn{2}{|c|}{$\begin{array}{l}\text { Wissens- } \\
\text { vernetzung }\end{array}$} \\
\hline & EG 1 & EG 2 & EG 1 & EG 2 & EG 1 & EG 2 & EG 1 & EG 2 & EG 1 & EG 2 & EG 1 & EG 2 \\
\hline $\begin{array}{l}\text { Problem- } \\
\text { definition }\end{array}$ & $\begin{array}{c}.31 \\
(.136)\end{array}$ & $\begin{array}{c}.20 \\
(.229)\end{array}$ & $\begin{array}{l}.16 \\
(.292)\end{array}$ & $\begin{array}{c}.23 \\
(.200)\end{array}$ & $\begin{array}{c}.28 \\
(.178)\end{array}$ & $\begin{array}{c}.52^{*} \\
(.032)\end{array}$ & $\begin{array}{l}.36 \\
(.109)\end{array}$ & $\begin{array}{c}.53^{*} \\
(.028)\end{array}$ & $\begin{array}{r}.49^{*} \\
(.047)\end{array}$ & $\begin{array}{c}.39 \\
(.082)\end{array}$ & $\begin{array}{c}.31 \\
(.136)\end{array}$ & $\begin{array}{c}.15 \\
(298)\end{array}$ \\
\hline $\begin{array}{l}\text { Probiem- } \\
\text { bearbeitung }\end{array}$ & $\begin{array}{c}.23 \\
(.200)\end{array}$ & $\begin{array}{c}.42 \\
(.048)\end{array}$ & $\begin{array}{l}-.14 \\
(.300)\end{array}$ & $\begin{array}{c}.39 \\
(.072)\end{array}$ & $\begin{array}{c}.13 \\
(.329)\end{array}$ & $\begin{array}{c}.63^{*} \\
(.012)\end{array}$ & $\begin{array}{c}.15 \\
(.295)\end{array}$ & $\begin{array}{c}40 \\
(.070)\end{array}$ & $\begin{array}{c}.33 \\
(.118)\end{array}$ & $\begin{array}{c}.32 \\
(.122)\end{array}$ & $\begin{array}{c}-.17 \\
(.264)\end{array}$ & $\begin{array}{c}.20 \\
(.232)\end{array}$ \\
\hline $\begin{array}{l}\text { Handlungs- } \\
\text { kontrolle }\end{array}$ & $\begin{array}{l}.26 \\
(.170)\end{array}$ & $\begin{array}{c}.31 \\
(.124)\end{array}$ & $\begin{array}{l}.17 \\
(.264)\end{array}$ & $\begin{array}{l}.44^{*} \\
(.048)\end{array}$ & $\begin{array}{c}.41 \\
(.074)\end{array}$ & $\begin{array}{l}.57^{\star} \\
(.020)\end{array}$ & $\begin{array}{c}.36 \\
(.097)\end{array}$ & $\begin{array}{c}.46^{\star} \\
(.046)\end{array}$ & $\begin{array}{c}.36 \\
(.097)\end{array}$ & $\begin{array}{c}.38 \\
(.084)\end{array}$ & $\begin{array}{l}.09 \\
(.375)\end{array}$ & $\begin{array}{c}.37 \\
.086)\end{array}$ \\
\hline
\end{tabular}

Einseilige Signifikanzlests, Angabe des Signifikanzniveaus in Kammern.

Für die Experimentalgruppe 1 (SoLe und Bilanzmethode) besteht lediglich ein signifikanter Zusammenhang zwischen der Häufigkeit der Problemdefinition und den Erfolgsaussichten der Problemlösung. Darüber hinaus zeigen sich tendenzielle Zusammenhänge (auf dem $10 \%$-Signifikanzniveau) zwischen der Häufigkeit der Handlungskontrolle und dem deklarativen Wissen, der Logik der Problemlösung und den Erfolgsaussichten. Im Unterschied hierzu fördern die durchgeführten Korrelationsanalysen für die beiden Lerngruppen der Experimentalgruppe 2 (SoLe und wirtschaftsinstrumentelles Rechnungswesen) eine Vielzaht überzufälliger Zusammenhänge zwischen den beobachtbaren Problemlöseaktivitäten und den Indikatoren für 
Lernerfolg zutage. Dies gilt sowohl für die Häufigkeit der Problemdefinition und der Problembearbeltung als auch für die Aktivitäten im Bereich Handlungskontrolle. Zudem sind die Korrelationen betragsmaßig höher als jene der Experimentalgruppe 1. Eine entsprechende Schüleraktivität im Unterricht scheint also insbesondere dann lernwirksam zu sein, wenn die Lerninhalte problemhaltig strukturiert sind und den Schülern eine vertiefte Auseinandersetzung mit der Materie erlauben, wie dies durch die Kombination des Selbstorganisierten Lernens mit dem wirtschaftsinstrumentellen Rechnungswesen angestrebt wird (siehe hierzu auch Seifried 2004b).

\subsubsection{Lehreraktivität in der Phase der Konteneinführung}

Schließlich ist zu prüfen, wie die Lehrpersonen agieren. Diesbezüglich besteht die Vermutung, dass der beim wirtschaftsinstrumentellen Rechnungswesen geforderte Rückbezug auf ein Modellunternehmen das Lehrerhandeln erleichtern sollte. Wie erinnerlich wird beim wirtschaftsinstrumentellen Rechnungswesen die Bilanz als didaktisches Referenzmodell durch das "Allgemeine Unternehmensmodell" ersetzt, anhand dessen Kosten- und Leistungsprozesse abgebildet und einer ökonomischen Interpretation zugeführt werden.

Die vermuteten Vorteile sollten ausdrücklich in der Phase der Konteneinführung hervortreten. Insbesondere während dieser Unterrichtsphase besteht die Möglichkeit, anhand dieses Modells die Auswirkungen von Geschäftsvorfällen auf einzelnen Bilanzpositionen darzustellen, wohingegen bei der Bilanzmethode der "Soll-an-HabenAlgorithmus" oder der Verweis auf ähnliche bzw. bereits bekannte Sachverhalte im Vordergrund stehen dürte. Im Zuge der Operationalisierung der Lehreraktivitaten unterscheiden wir demzufolge drei Kategorien:

(1) Rückbezug auf den Soll-an-Haben-Algorithmus/Merksatz (Bsp.: „Das Bankkonto nimmt zu, somit ist die Sollseite des Kontos betroffen, da es sich bei dem Konto Bank um ein Aktivkonto handelt und bei diesen die Mehrungen im Soll gebucht werden."),

(2) Rückbezug auf einen bereits bekannten Sachverhalt (Bsp.: [...] bei dem Konto Kasse wird genauso vorgegangen wie beim Konto Bank) sowie

(3) „Rückbezug auf das Unternehmensmodell (Bsp.: „Die Überweisung an den Lieferanten wird hier am Untermehmensmodell durch den Wertefluss vom Konto Bank zum Lieferanten verdeuticht. ").
Erwartungsgemaß zeigt sich, dass bei der Bilanzmethode die Verwendung von Merksätzen überwiegt (überwiegen muss!), wohingegen bei einer Ausrichtung der Lehr-Lern-Prozesse an der Didaktik des wirtschaftsinstrumentellen Rechnungswesens der Rückbezug auf das Unternehmensmodell die bedeutendste Variante darstellt (siehe Abbildung 6). Hălt man sich die Befunde zur Lernleistung vor Augen (s.o.), so wird deutlich, dass diese Vorgehensweise offenbar mit Vorteilen für Lernende (bessere Lernergebnisse, bessere emotionale Befindlichkeit) und für Lehrende (Erweiterung des Handlungspotenzials in lehrerzentrierten Unterrichtsphasen) verbunden ist.

Abb. 6: Lehrerverhalten bei der Konteneinführung

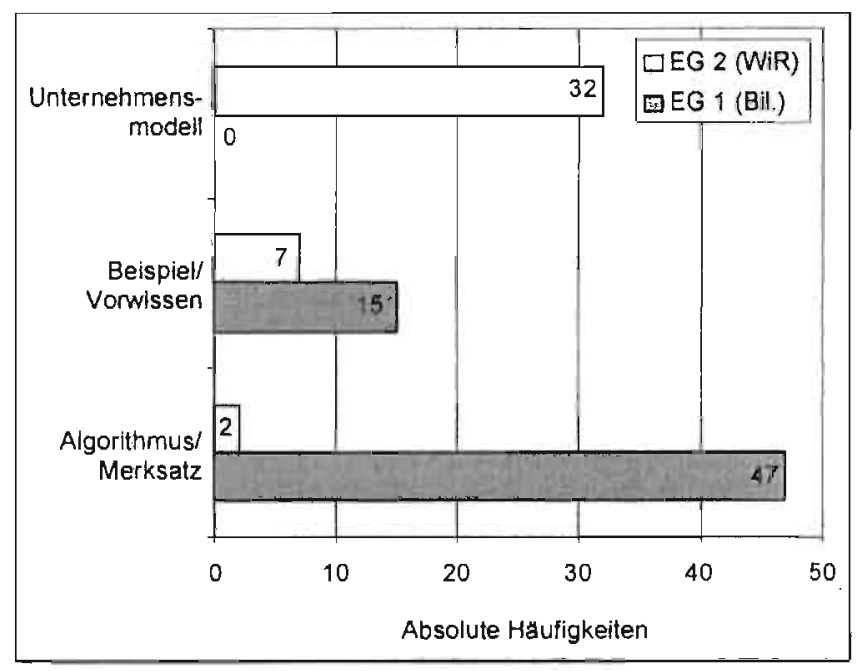

\section{Implikationen für die Unterrichtspraxis}

Die in diesem Beitrag vorgestellten theoretischen Überlegungen und empirischen Befunde helfen bei der Beantwortung dreier Fragestellungen weiter, die im Mittelpunkt der fachdidaktischen Auseinandersetzung mit dem Rechnungswesenunterricht stehen:

- Mit welchem Ziel sollen sich Lemende mit dem Rechnungswesen beschäftigen? Bei der Gestaltung einer selbstorganisationsoffenen Lernumgebung für das Rechnungswesen hatten wir das Leitziel der Förderung der ökonomischen Kompetenz bei gleichzeitiger Beherrschung der Verfahrenstechniken angestrebt. Dabei ist der Kom- 
petenzbegriff als komplexes Kriterienbündel zu verstehen und umfassend zu interpretieren. Ein ganzheitliches Begriffsverständnis schließt neben der Vermittlung und dem Erwerb von ökonomischem Wissen, das sowohl im Unternehmens- und Verwaltungsbereich als auch im privaten Kontext zielgerichtet und effektiv eingesetzt werden kann, auch emotional-motivationale Aspekte und übergreifende Fähigkeiten wie komplexes Problemlösen, Kommunikationsfähigkeit oder Teamfähigkeit ein (Sembill 1992a; Achtenhagen 1996).

- Welche Inhalte sollen dem Lerngebiet Rechnurigswesen zugeordnet werden und wie soll die Sequenzierung dieser Inhalte aussehen?

Die Umsetzung dieser Zielsetzung erfordert eine fachdidaktische Konzeption, die verstärkt den Stellenwert der Buchführung als Informationsinstrument in den Mittelpunkt rückt. Im Unterricht ist klar herauszuarbeiten, wie betriebswirtschaftliche Sachverhalte in einem Informationssystem zu erfassen sind, welche Auswertungen ein Informationssystem bereitstellen kann und wie Entscheidungen durch die Gestaltung des Informationssystems beeinflusst werden. Mit der Didaktik des wirtschaftsinstrumentellen Rechnungswesens liegt eine Konzeption vor, die sowohl inhaltlich als auch - mit Abstrichen - methodisch sehr viel stärker als die in Fachkreisen bisher diskutierten Entwüre auf den Aufbau ökonomischer Kompetenz im oben skizzierten Sinne ausgerichtet ist.

- Wie ist der Rechnungswesenunterricht aus methodischer Sicht zu gestalten? Angesichts der unterrichtsmethodischen Problemlagen des herkömmlichen Rechnungswesenunterrichts liegt es auf der Hand, dass die Diskussion möglicher Ansatzpunkte zur Steigerung der Unterrichtsqualität über den Aspekt der Bestimmung, Begründung und Sequenzierung der Ziele und Inhalte des Unterrichts hinaus die Auseinandersetzung mit methodisch-medialen Fragestellungen umfassen muss. Dabei ist eine konsequente Ausweitung der Handlungsorientierung zu fordern. Damit ist nicht gemeint, im herkömmlichen Unterricht hier und da eine Gruppenarbeitsphase einzustreuen oder Schüler in irgendeiner Form praktische Tătigkeiten durchführen zu lassen, ohne dass sich aus diesen Tätigkeiten ein vertieftes ökonomisches Verständnis entwickelt. Diese falsch verstandene Interpretation des Terminus "Handlungsorientierung" - Achtenhagen (1994) spricht in diesem Zusammenhang von "Wuseldidaktik" dürfte nur in seltenen Fällen zu den erwünschten Ergebnissen führen.

Im Unterschied hierzu sind die Lehrpersonen beim Selbstorganisierten Lernen aufgefordert, Lehr-Lern-Prozesse ganzheitlich zu betrachten und den Lerner konsequent in das Zentrum der pädagogischen Bemühungen zu rücken. Dies erfordert eine stringente Vorgehensweise auf den verschiedenen Ebenen der Curriculum- und Unterrichtsgestaltung (Dubs 2001): Auf der Makroebene (normative Ebene) gilt es zunächst zu überdenken, welche Ziele mit Schule und Unterricht erreicht werden sollen. In diesem Zusammenhang muss beispielsweise diskutiert werden, in welchem Ausmaß Lernende bei der Planung, Durchführung und Kontrolle der Lehr-LernSituationen zu beteiligen sind. Diesbezüglich ist zu fordern, dass diese Entscheidung zugunsten einer umfassenden Selbstbestimmung und Teilhabe der Lernenden auf allen drei Unterrichtsebenen (einschließlich der Kontroll- und Bewertungsdimension) zu treffen ist. Auf der Mesoebene (curricular-didaktische Ebene) sind dann im Zuge der Lehrplangestaltung Freiräume zu schaffen, damit Lehr-Lern-Prozesse initiiert werden können, die offen sind für selbstständiges Lernen, und auf der Mikroebene (instruktionale Ebene) schließlich geht es darum, wie Lehr-Lern-Situationen konkret zu gestalten sind, um erfolgreiche Lehr-Lern-Prozesse initiieren zu können. Hier gilt es, die Kompatibilität fachdidaktisch-methodischer und fachdidaktisch-curricularer Modellierungen sicherzustellen. Auf den Rechnungswesenunterricht angewandt bedeutet dieser pädagogische Allgemeinplatz, dass weder die alleinige Beschäftigung mit der Auswahl und Sequenzierung der Lerninhalte noch die ausschließliche Fokussierung auf unterrichtsmethodische Aspekte zielführend sein dürfte. Vielmehr bedarf es einer ganzheitlichen Betrachtungsweise, will man wirklich die herrschenden Unterrichts- und Begründungsmuster durchbrechen und eine Ausweitung der Handlungsorientierung realisieren, denn abstrakte, wenig anschauliche und hochformalisierte Lerninhalte "zwingen" die Lehrpersonen zu einer lehrerzentrierten Vorgehensweise.

Abschließend stellt sich die Frage, wie die gewonnenen Erkenntnisse für das universitäre Lehrangebot der Ausbildung zum Diplom-Handelslehrer nutzbar gemacht werden können. Hierzu wurde ein virtuelles Seminar "Planung und Vorbereitung eines selbstorganisationsoffenen Unterrichts am Beispiel Rechnungswesen" konzipiert, das über die Virtuelle Hochschule Bayern (http://www.vhb.org) abgerufen werden kann (siehe hierzu den Beitrag von Wolf, Seifried \& Stădler in diesem Band). 


\section{Literatur}

Achtenhagen, F. (Hrsg.) (1990): Didaktik des Rechnungswesens. Programm und Kritik eines wirtschaftsinstrumentellen Ansatzes, Wiesbaden: Gabler.

Achtenhagen, F. (1994): Curriculumentwicklung unter dem Aspekt Handlungsorientierung, in: Landesinstitul für Schule und Weiterbildung ( $\mathrm{Hrsg}$ ): Curriculumentwicklung für berufsbildende Schulen, Soest, S. 57-108.

Achtenhagen, F. (1996): Entwicklung ökonomischer Kompetenz als Zieikategorie des Rechnungswesenunterrichts, in: PreißB, P. \& Tramm, T. (Hrsg.): Rechnungswesenunterricht und ökonomisches Denken. Dídaktische Innovationen für die kaufmännische Ausbildung, Wiesbaden: Gabier, S. 22-44.

Baumert, J., Bos, W. \& Lehmann, R. (Hrsg.) (2000): TIMSS/III. Dritte Internationale Mathematik- und Naturwissenschaftsstudie. Mathematische und naturwissenschaftliche Bildung am Ende der Schullaufbahn, Band 2: Mathematische und physikalische Kompetenzen am Ende der gymnasialen Oberstufe, Opladen: Leske + Budrich

Baumert, J. \& Köller, O. (2000): Unterrichtsgestaltung, verständnisvolles Lernen und multiple Zielerreichung Im Mathematik- und Physikunterricht der gymnasialen Oberstufe, in: Baumert, J., Bos, W. \& Lehmann, R. (Hrsg.) (2000): TIMSS/III. Dritte Internationale Mathematik- und Naturwissenschaftsstudie. Mathematische und natunwissenschaftliche Bildung am Ende der gymnasialen Oberstufe Opladen: Leske + Budrich, S. 271-315.

Baumert, J., Klieme, E., Neubrand, M., Prenzel, M., Schiefele, U., Schneider, W., Stanat, P., Tillmann, K.J. \& Weiss, M. (2001): PISA 2000. Basiskompetenzen von Schülerinnen und Schülern im internationalen Vergleich, Opladen: Leske + Budrich.

Beyen, W. (2003): Von der handlungsorientierten zur konstruktivistischen Perspektive? Überlegungen zur methodisch-konzeptionellen Gestaltung des WirtschaftslehreUnterrichts, in: Zeitschrift für Berufs- und Wirtschaftspädagogik, 99. Jg., H. 1, S. 107-125.

Dann H.-D., Diegrilz, T. \& Rosenbusch, H. S. (2002): Gruppenunterricht im Schulalltag: Ergebnisse eines Forschungsprojekts und praktische Konsequenzen, in: Pädagogik, 54. Jg., H. 1, S. 11-14.

Dubs, R. (2001): Mehr Klarheit für die Unterrichtspraxis - einige kritische Anmerkungen, in: Zeitschrift für Berufs- und Wirtschaftspädagogik, 97 . Jg., H. 1, S. 1-5.

Friedrich, G. \& Preiß, G. (2003): Neurodidaktik. Bausteine für eine Brückenbildung zwischen Hirnforschung und Didaktik, in: Pädagogische Rundschau, 57. Jg., H. 2., S. 181-199

Gruber, H., Mandl, H. \& Renkl, A. (2000): Was lemen wir in Schule und Hochschule: Träges Wissen?, in: Mandl, H. \& Gerstenmaier, J. (Hrsg.): Die Kluft zwischen Wissen und Handeln, Göttingen, Bern, Toronto \& Seattle: Hogrefe, S. 139-156.

Hacker, W. (1992): Expertenkönnen, Göttingen, Bern, Toronto \& Seattle: Verlag für angewandte Psychologie.
Mayer, R. E. \& Wittrock, M. C. (1996): Problem-solving transfer, in: Berliner, D. C. \& Calfee R. C (Eds.): Handbook of educational psychology, New York (NY): Macmillan, pp. 47-62.

Pätzold, G., Klusmeyer, J., Wingels, J. \& Lang, M. (2003): Lehr-Lern-Methoden in der beruflichen Bildung. Eine empirische Untersuchung in ausgewathlten Berufsfeldern, Oldenburg: Universität Oldenburg.

Preiß, P. (1999): Didaktik des wirtschaftsinstrumentellen Rechnungswesens, München \& Wien: Oldenbourg.

Preiß, P. \& Tramm, T. (1996): Die Göttinger Unterrichtskonzeption des wirtschaftsinstrumentellen Rechnungswesens, in: Preiß, P. \& Tramm, T. (Hrsg.): Rechnungswesenunterrich und ökonomisches Denken. Didaktische Innovationen für die kaufmännische Ausbildung, Wiesbaden: Gabler, S. 222-323.

Petri, G. (1991): Idee, Realităt und Entwicklungsmöglichkeiten des Projektlernens, Arbeitsberichte Reihe IIINr. 22, Graz 1991.

Prenzel, M. (1994): Fragebogen zu „Motivationalen Bedingungen" und zu "Motivationalen Prozessen beim Lernen", Regensburg.

Reinisch, H. \& Struve, K. (2002): Was können wir aus der Geschichte beruflicher Arbeit unıd berufbezogener Didaktik lernen? Zur Bedeutung einer historischen Analyse/Synthese der gegenständlichen Dimension ökonomischer und gewerblich-lechnischer Bildung in: $E$ geger. Horlebein. M L Lisop, I. Reinisch, H. \& Tramm, T. (Hrsg.): Bilanzierungen Schulentwicklung, Lehrerbildung und Wissenschaftsgeschichte im Feld der Wirtschafts und Berufspädagogik, Frankfurt/Main: GAFB, S. 99-150.

Renkl, A., Gruber, H. \& Mandl, H. (1996): Kooperatives, problemorientiertes Lernen in der Hochschule, in: Lompscher, J. \& Mandl, H. (Hrsg.): Lehr- und Lernprobleme im Studium Bedingungen und Veränderungsmöglichkeiten, Bern: Huber, S. 131-147.

Schneider, W. (2000): Didaktik des Rechnungswesens im Konflikt zwischen Fachwissenschaft, komplexer Realität und subjektiver Faßlichkeit, in: Euler, D., Jongebloed, H.-C. \& Sloane, P. F. E. (Hrsg.): Sozialökonomische Theorie - sozialökonomisches Handeln. Konturen und Perspektiven der Wirtschafts- und Sozialpädagogik, Kiel: bajOsch-Hein Verlag für Berufs- und Wirtschaftspädagogik, S. 191-210.

Seeber, S. (2000): Stand und Perspektiven von Bildungscontrolling, in: Seeber; S., Krekel, E M. \& Buer, J. van (Hrsg.). Bildungscontrolling - Ansätze und kritische Diskussion zur EffiM. \& Buer, J. van (Hrsg.), Bildungscontrolling - Ansätze und
zienz von Bildung, Frankfurt/Main u.a.: Peter Lang, S. 19-50.

Seifried, J. (2002): Selbstorganisiertes Lernen im Rechnungswesen, in: Zeitschrift für Berufsund Wirtschaftspädagogik, 98. Jg., H.1, S. 104-121.

Seifried, J. (2004a): Fachdidaktische Variationen in einer selbstorganisationsoffenen Lernumgebung - Eine empirische Untersuchung des Rechnungswesenunterrichts, Wiesbaden: Deutscher Universitäts-Verlag.

Seifried J (2004b): Schüleraktivităten beim selbstorganisierten Lernen und deren Auswir-

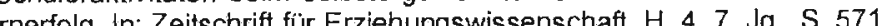
586 . 
Sembill, D. (1992a): Problemlösefähigkeit, Handlungskompetenz und Emotionale Befindlichkeit. Zielgrößen Forschenden Lernens, Göttingen, Toronto \& Zürich: Hogrefe.

Sembill, D. (1992b): Handlungs- und Emotionsstrukturen. Operationalisierungen, Rekonstruktion und Ergebnisse. Dokumentationsband zur Monographie: Problemlösefähigkeit, Handlungskompetenz und Emotionale Befindlichkeit. Zielgrößen Forschenden Lernens, Göttingen \& Mannheim.

Sembill, D. (1999): Selbstorganisation ats Modellierungs-, Gestaltungs- und Erforschungsidee beruflichen Lernens, in: Tramm, T., Sembill, D., Klauser, F. \& John, E. G. (Hrsg.): Professionalisierung kaufmännischer Berufsbildung: Beiträge zur Offnung der Wintschaftspädagogik für die Anforderungen des 21. Jahrhunderts. Festschrift zum $60 . \mathrm{Ge}-$ burtstag von Frank Achtenhagen. Frankfurt/Main u.a.: Lang, S. 146-174.

Sembill, D. (2000): Selbstorganisiertes und Lebenslanges Lernen, in: Achtenhagen, F. \& Lempert, $W$. (Hrsg.): Lebenstanges Lernen - seine Grundlegung im Kindes- und Jugendalter, Band 4: Formen und Inhalle von Lemprozessen, Opladen: Leske + Budrich, S. 6090.

Sembill, D. (2003a): Results of self-organized learning in vocational education, in: Achtenhagen, F. \& John, E. G. (Eds.); Milestones of vocational and occupational education and training, Vol. 1: The teaching-learning perspective, Bielefeld: Bertelsmann, pp. 81-106.

Sembill, D. (2003b): Emotionale Befindlichkeit als bestimmende und sinngebende Voraussetzung von Lern- und Lebenswirklichkeit, in: van Buer, J. \& Zlatkin-Troitschanskaia, O. (Hrsg.): Berufliche Bildung auf dem Prüfstand - Entwicklung zwischen systemischer Steuerung. Transformalion durch Modellversuche und unterrichtlicher Innovation. Frankfurt/Main: Peler Lang. S. 181-205.

Sembill, D. (2004): Abschlussbericht zu „Prozessanalysen Selbstorganisierten Lernens" im Rahmen des DFG-Schwerpunktprogramms "Lehr-Lern-Prozesse in der kaufmännischen Erstausbildung". Bamberg. Download unter hitp://wipaed.sowi.uni-bamberg.de/awgs/ wipaed2002/download-pdf/abschlussbericht_prozess-analysen.pdf

Sembill, D., Wolf, K. D., Wuttke, E., Santjer, I. \& Schumacher, L. (1998): Prozessanalysen Selbstorganisierten Lernens, in: Beck, K. \& Dubs, R. (Hrsg.): Kompetenzerwerb in der Berufserzlehung. Kognitive, motivationale und moralische Dimensionen kaufmännischer Qualifizierungsprozesse, in: Zeitschrift für Berufs- und Wirtschaftspädagogik, Beiheft Nr. 14, Stultgart: Franz Steiner, S. 57-79.

Sembill, D., Schumacher, L., Wolf, K. D. Wuttke, E. \& Santjer-Schnabel, I. (2001): Förderung der Problemlösefähigkeit und der Motivation durch Selbstorganisiertes Lernen, in: Beck, K. \& Krumm, V. (Hrsg.): Lehren und Lernen in der beruflichen Erstausbildung. Grundlagen einer modernen kaufmănnischen Berufsqualifizierung, Opladen: Leske + Budrich, S. 257-281.

Sloane, P. F. E. (1996): Didaktik des Rechnungswesens, Pfaffenweiler: CentaurusVerlagsgeseilschaft.

Wuttke, E. (1999): Motivation und Lernstrategien in einer selbstorganisationsoffenen Lernumgebung. Eine empirische Untersuchung bei Industriekaufleuten, Frankfurt/Main u.a. Lang. 